\title{
NATIONAL TAIWAN UNIVERSITY RADIOCARBON DATES III
}

\section{TSUNG-KWEI LIU}

Department of Geology, National Taiwan University, 245 Choushan Road, Taipei, Taiwan Republic of China

\section{INTRODUCTION}

This list consists of radiocarbon dates for geological samples measured from 1988 to 1990 at the resumed National Taiwan University (NTU) Radiocarbon Dating Laboratory. The NTU Radiocarbon Dating Laboratory was re-activated in 1988, after more than 14 years.

The NTU laboratory uses an LKB Quantulus $1220^{\mathrm{TM}}$ for benzene liquid scintillation counting and primarily follows laboratory procedures described by Coleman (1973). All ages are calculated on the basis of a ${ }^{14} \mathrm{C}$ half-life of $5568 \mathrm{yr}$, using the NBS Oxalic Acid II standard as reference. Dates are expressed in ${ }^{14} \mathrm{C}$ yr relative to $\mathrm{AD} 1950$ and corrected for isotopic fractionation $\left(\delta^{13} \mathrm{C}\right.$ values are relative to PDB).

Errors (one standard deviation, or $1 \sigma$ ) quoted with dates account only for uncertainties in counting statistics. Ages younger than $200 \mathrm{yr}$ are reported as modern. Percentage of modern is reported only if $\Delta^{14} \mathrm{C}$ is greater than zero. Assignment of minimum ages is based on $2 \sigma$ criteria. If the difference in activity between the sample and the background is $<2 \sigma$ of the difference, the minimum age reported is based on the net activity plus $2 \sigma$, as suggested in Stuiver and Polach (1977).

Except as otherwise indicated, wood, peat and charcoal samples are pretreated by boiling with dilute $\mathrm{NaOH}$ and dilute $\mathrm{HCl}$ before combustion to $\mathrm{CO}_{2}$ and final conversion to liquid benzene for counting. Carbonaceous clays are pretreated with either dilute $\mathrm{HCl}$ only or $\mathrm{HCl}$ and cool dilute $\mathrm{NaOH}$, depending on whether or not the carbonaceous matter is totally decomposed by dilute $\mathrm{NaOH}$. Carbonate samples are generally pretreated by dissolving away the outer portion with dilute $\mathrm{HCl}$.

Sample descriptions are based on information provided by the sample submitters.

\section{ACKNOWLEDGMENTS}

This research was financially supported by National Science Council grant NSC81-0202-M-002-01. I thank Drs. Dennis D. Coleman and Jack C. L. Liu of the Illinois State Geological Survey for their help in setting up the vacuum line for benzene synthesis. This paper benefited from the comments of an anonymous reviewer.

\section{Geological SAMPLeS}

\section{Taiwan}

\section{Taipei City}

Samples from Taipei City $\left(25^{\circ} 01-03^{\prime} \mathrm{N}, 121^{\circ} 30-36^{\prime} \mathrm{E}\right)$. Collected 1989 to 1990 and submitted by T.-K. Liu. 
NTU-1093. TPS1045

$$
\begin{aligned}
5060 \pm 60 \\
\delta^{13} C=-23.0 \% 0 \\
4215 \pm 50 \\
\delta^{13} C=-27.9 \% 0 \\
>41,000 \\
\delta^{13} C=-30.6 \% 0
\end{aligned}
$$

Wood from Taipei Railroad Station, at $10.7 \mathrm{~m}$ depth.

\section{NTU-1113. TPS771218}

Wood from silt zone, at $10 \mathrm{~m}$ depth.

NTU-1163. FTSUI-02

Wood from river terrace deposits.

NTU-1218. 761213C

$\mathbf{7 7 3 0} \pm \mathbf{5 0}$ $\delta^{13} C=-0.6 \%$

\section{NTU-1222. TPS-761218}

$6600 \pm 60$

Woody materials from construction foundation, $11.3 \mathrm{~m}$ below surface.

NTU-1223. TPS-WTB

Oyster shell, $\sim 11 \mathrm{~m}$ below surface.

NTU-1232. TPS-7610174A

Shell, $7.5 \mathrm{~m}$ below surface.

NTU-1233. TPS-761208A

Shell, $9.5 \mathrm{~m}$ below surface.

$8040 \pm 50$

NTU-1240. TPS-7610111B

Wood, $17.4 \mathrm{~m}$ below ground surface.

NTU-1251. TPS-780814A

$\delta^{13} C=-28.3 \%$

Wood, $40.1 \mathrm{~m}$ below surface, at the bottom of the Sungshan Formation.

Comment (T.-K.L.): Results are consistent with the stratigraphic sequence. Thus, the Sungshan Formation is of well-established Holocene age.

\section{Taipei County}

\section{Hsinchuang Village Series}

Wood from Hsinchuang Village, Taipei County $\left(25^{\circ} 02^{\prime} \mathrm{N}, 121^{\circ} 26^{\prime} \mathrm{E}\right)$, from a silt zone $5 \mathrm{~m}$ below surface. Collected 1989 and submitted by T.-K. Liu.

NTU-1115. TPS771216A

$$
\begin{array}{r}
4400 \pm 50 \\
\delta^{13} C=-28.5 \% 0
\end{array}
$$


NTU-1114. TPS-771216B

$$
\begin{array}{r}
4310 \pm 50 \\
\delta^{13} C=-27.8 \%
\end{array}
$$

Comment (T.-K.L.): Dates are for a fossil forest, which was found in the Taipei basin.

\section{Chinshan Village Series}

Carbonized wood from Chinshan Village, Taipei County $\left(25^{\circ} 02^{\prime} \mathrm{N}, 121^{\circ} 37^{\prime} \mathrm{E}\right)$, near the surface of marine terraces. Collected 1989 and submitted by Y. H. Lou and T.-K. Liu, National Taiwan University.

NTU-1110. Punshui

$$
\delta^{13} C=-21.8 \% 0
$$

NTU-1111. Tiaoshi

$3020 \pm 40$

$\delta^{13} C=-30.8 \%$

NTU-1137. Chinshan

$2360 \pm 40$

$\delta^{13} C=-31.3 \%$

Comment (T.-K.L.): NTU-1110 was collected from $9.7 \mathrm{~m}$ and NTU-1111 from $14.5 \mathrm{~m}$ above sea level (asl), respectively.

\section{Shihmen Village Series}

Samples from a marine terrace in Shihmen Village, Taipei County $\left(25^{\circ} 16^{\prime} \mathrm{N}, 121^{\circ} 36^{\prime} \mathrm{E}\right)$. Collected 1989 and submitted by Y. H. Lou and T.-K. Liu.

NTU-1131. Tsaoli 1

Wood from the upper portion of silty clay.

NTU-1132. Tsaoli 2

Charcoal from the lower portion of marine terrace.

Comment (T.-K.L.): Dates confirm a disconformity between Tsaoli 1 and 2; Tsaoli 2 looks like lignite and is expected to be much older.

\section{Tanshui Village Series}

Carbonized plant detritus from Tanshui Village, Taipei County $\left(25^{\circ} 18^{\prime} \mathrm{N}, 121^{\circ} 28^{\prime} \mathrm{E}\right)$. Collected 1989 and submitted by Y. H. Lou and T.-K. Liu.

NTU-1117. NPT1130C

From a clay zone, $8 \mathrm{~m}$ below surface.

\section{NTU-1118. NTP30B}

From a clay zone, $7.2 \mathrm{~m}$ below surface.

$$
\begin{array}{r}
3110 \pm 40 \\
\delta^{13} C=-29.0 \% 0 \\
\\
>47,000 \\
\delta^{13} C=-25.0 \% 0
\end{array}
$$

Comment (T.-K.L.): Samples are from emerged marine deposits. NPT1130C is from a lower position, $\sim 14 \mathrm{~m}$ asl, and associated with a brackish shell, Placuna placenta. 


\section{Shulin Village Series}

Samples are from Shulin Village, Taipei County $\left(25^{\circ} 18^{\prime} \mathrm{N}, 121^{\circ} 28^{\prime} \mathrm{E}\right)$.

NTU-1081. SL508G3

$37,010 \pm 500$

Carbonized tree trunk in upright living position.

NTU-1070. SL770508

$30,510 \pm 250$

$\delta^{13} C=-24.7 \%$

Wood from a sandy layer in river terrace deposits. Collected 1988 and submitted by Y. K. Chen and T.-K. Liu.

Comment (T.-K.L.): Dates are for a buried fossil forest on southwest border of the Taipei basin.

\section{Juifang Village Series}

Wood from Juifang Village, Taipei County $\left(25^{\circ} 08^{\prime} \mathrm{N}, 121^{\circ} 51^{\prime} \mathrm{E}\right)$, from a gray clay zone in a coastal tableland $\sim 60 \mathrm{~m}$ asl. Collected 1988 and submitted by C. M. Hsu, National Taiwan Oceanography University.

NTU-1073. Kengzliao 88021

$37,140 \pm 500$

$\sim 2.0 \mathrm{~m}$ depth.

$\delta^{13} C=-25.5 \%$

NTU-1083. Kengzliao 88022

$43,160 \pm 1040$

$\sim 2.5 \mathrm{~m}$ depth.

$\delta^{13} C=-25.0 \%$

NTU-1094. Kengzliao 88023

$44,800 \pm 1300$

$\sim 2.5 \mathrm{~m}$ depth.

$\delta^{13} C=-27.0 \%$

Comment (C.M.H.): Dates are as expected and they agree with the stratigraphic sequence.

\section{Taitung County}

Fukang Series

Samples from Fukang Village, Taitung County $\left(22^{\circ} 48-52^{\prime} \mathrm{N}, 121^{\circ} 11-12^{\prime} \mathrm{E}\right)$. Collected $1988-1989$ by T.-K. Liu, C. H. Wang, and M. L. Hsieh, National Taiwan University.

NTU-1059. Sinchiao 0227L

$8700 \pm 70$

$\delta^{13} \mathrm{C}=-0.8 \%$

Mollusks from beach sand of a marine terrace $\sim 45 \mathrm{~m}$ asl, $11.5 \mathrm{~m}$ below surface.

NTU-1069. Heifachiao 0227P

$2820 \pm 50$

$\delta^{13} \mathrm{C}=+1.3 \%$

Shell from beach sand of a marine terrace $\sim 40 \mathrm{~m}$ asl. Associated with pottery fragments. 
NTU-1082. Fukang 0227R

$3630 \pm 50$

$\delta^{13} C=-1.0 \%$

Coral (aragonite >95\%) from the 0.5 -m-thick coral reef unconformably overlying a wave-cut sandstone marine terrace, $40 \mathrm{~m}$ asl. Collected 1988 and submitted by T.-K. Liu.

Comment (T.-K.L.): Represents the dominant Terrace II of the Coastal Range in eastern Taiwan.

\section{NTU-1129. Fukang 1}

Fresh coral (aragonite >95\%), $\sim 30 \mathrm{~m}$ asl.

$3090 \pm 40$

$\delta^{13} C=-1.2 \%$

NTU-1189. Fukang 2

$1660 \pm 40$

Coral (aragonite $>95 \%$ ) from recent nearshore terrace, $\sim 2 \mathrm{~m}$ asl.

NTU-1224. GP78-8

$3580 \pm 40$

$\delta^{13} C=-1.8 \%$

Coral (aragonite >95\%) from the lower portion of the reef overlying sandstone. Collected from the same reef layer as NTU-1082.

Comment (M.L.H.): Dates are as expected.

\section{Chengkung Village Series}

Samples from Chengkung Village, Taitung County $\left(22.8-23.1^{\circ} \mathrm{N}, 121.1-121.4^{\circ} \mathrm{E}\right)$. Collected 1988 and submitted by C. H. Wang, Institute of Earth Science, Academia Sinica, T.-K. Liu and M. Y. Hsu, National Taiwan Normal University.

NTU-1068. 7712-0914

$1920 \pm 50$

Coral, $\sim 1 \mathrm{~m}$ asl.

NTU-1080. 7713-1131

$1500 \pm 50$

Coral from the coastal reef, $\sim 1 \mathrm{~m}$ asl.

NTU-1106. CK0227J

$3380 \pm 40$

Coral from $2 \mathrm{~m}$ below the surface of the coastal reef $\sim 15 \mathrm{~m}$ asl.

$\delta^{13} C=-0.1 \%$

Comment (C.H.W.): Samples were in-situ. Recrystallization is insignificant.

NTU-1127. 770227P

$3490 \pm 50$

Coral (aragonite $>95 \%$ ) from the reef terrace, $\sim 35 \mathrm{~m}$ asl.

$\delta^{13} C=-0.3 \%$

NTU-1187. 770227K

$5550 \pm 50$

Coral from the reef terrace.

Comment (T.-K.L.): Dates are for Terrace II of the Coastal Range in eastern Taiwan.

\section{NTU-1174. GP78-4}

Shell from the sea cave bottom, in the Takangkou Formation.
$1340 \pm 40$

$\delta^{13} C=+1.78 \%$ 
NTU-1213. GP78-5

Mollusks from the marine terrace.

\section{NTU-1225. GP78-6}

$3430 \pm 40$

Coral from the marine terrace.

NTU-1237. GP78-7

Coral from the top of the marine terrace.

Comment (M.Y.H.): Results of NTU-1174 and -1237 are as expected; NTU-1225 is $\sim 1000$ yr older than expected, suggesting a lower uplift rate. NTU-1213 is not in situ.

\section{NTU-1142. HS003}

$950 \pm 40$

$\delta^{13} \mathrm{C}=-28.1 \%$

Wood from a sandy beach on top of the coral zone. Collected 1989 and submitted by M. L. Hsieh.

Comment (M.L.H.): Age is as expected.

\section{Changpin Village Series}

Samples from Changpin Village, Taitung County $\left(25^{\circ} 20^{\prime} \mathrm{N}, 121^{\circ} 25^{\prime} \mathrm{E}\right)$. Collected 1989 and submitted by M. L. Hsieh.

NTU-1122. HS002

Wood from a mud zone.

NTU-1098. LPM-CB

Wood from a mud and conglomerate zone.

NTU-1188. HSO04

Shell from a gully of the shore platform, $17 \mathrm{~m}$ asl.

NTU-1230. HS005

Wood from nearshore muddy deposits.

NTU-1247. HSO07

Wood from offshore muddy deposits.

NTU-1253. HS010

Wood from offshore muddy deposits.

Comment (M.L.H.): Ages are in an expected range.

\section{Huatung Series}

Carbonates from the eastern coast of Taiwan. Collected 1989 and submitted by J. C. Lin and P. M. Liew, National Taiwan University.
$5070 \pm 50$

$\delta^{13} C=-25.0 \%$

$5130 \pm 60$

$\delta^{13} C=-27.9 \%$

$$
\begin{array}{r}
1970 \pm 40 \\
\delta^{13} C=+2.0 \%
\end{array}
$$

$\mathbf{7 8 2 0} \pm \mathbf{5 0}$

$\delta^{13} C=-28.8 \%$

1050 \& 40

$\delta^{13} C=-27.3 \%$

$5230 \pm 30$

$\delta^{13} C=-25.0 \%$ 
NTU-1242. PM-1

\section{NTU-1268. PM-2}

NTU-1272. PM-3
$4650 \pm 40$

$\delta^{13} \mathrm{C}=+3.7 \%$

$2520 \pm 50$

$\delta^{13} \mathrm{C}=+2.1 \%$

$\mathbf{3 2 1 0} \pm \mathbf{5 0}$

$\delta^{13} \mathrm{C}=+1.9 \%$

Reef limestones from Holocene deposits.

Comment (P.M.L.): Dates are as expected.

\section{Lanyü Series}

Samples from Lanyü, Taitung County $\left(22^{\circ} 10-30^{\prime} \mathrm{N}, 121^{\circ} 20-35^{\prime} \mathrm{E}\right)$. Collected 1987 to 1989 and submitted by $\mathrm{C}$. $\mathrm{H}$. Wang and $\mathrm{Y}$. K. Chen.

\section{NTU-1053. LY76-02}

Fresh coral (aragonite $>95 \%$ ), $\sim 2 \mathrm{~m}$ asl.

\section{NTU-1057. LY76-02}

Fresh coral (aragonite $>95 \%$ ), $\sim 2 \mathrm{~m}$ asl.

NTU-1091. 7702-1630

Coral from reef, $\sim 7 \mathrm{~m}$ asl. Very light yellow in color.

\section{NTU-1096. 7704-1025}

Fresh coral (aragonite $>95 \%$ ), $2 \mathrm{~m}$ asl.

NTU-1170. 7706-1520

Coral (aragonite $>95 \%$ ) from a nearshore terrace, $\sim 3 \mathrm{~m}$ asl.

$$
\begin{array}{r}
3600 \pm 60 \\
\delta^{13} C=-0.7 \% 0
\end{array}
$$

$3370 \pm 50$ $\delta^{13} C=-0.9 \%$

$27,300 \pm 250$ $\delta^{13} \mathrm{C}=-1.2 \%$
$3630 \pm 50$ $\delta^{13} C=-0.1 \%$

$6690 \pm 60$ $\delta^{13} C=-0.4 \%$

Comment (C.H.W.): Samples were collected from $2-7 \mathrm{~m}$ asl, in living position and show good preservation.

NTU-1226. LY781009A

Algal limestone from a sea cave.

NTU-1227. LY781009B

Coral from a reef (aragonite $>95 \%$ ).

NTU-1229. LY781010A

Algal limestone (calcite $>95 \%$ ) from the top of a cliff, $15 \mathrm{~m}$ asl.

NTU-1234. LY781010B

Coral from a reef. $\mathbf{4 0 3 0} \pm \mathbf{4 0}$ $\delta^{13} C=+1.6 \%$

$$
\begin{array}{r}
4120 \pm 40 \\
\delta^{13} C=-0.6 \% 0
\end{array}
$$

$31,900 \pm 300$ $\delta^{13} C=-0.5 \%$

$4150 \pm 40$ $\delta^{13} C=+0.4 \%$ 
Comment (Y.K.C.): Three younger dates for marine Terrace I. NTU-1229 for Terrace II, corresponds with NTU-1107 from the Lutao series, below.

\section{Lutao Series}

Samples are from Lutao, Taitung County $\left(22^{\circ} 30^{\prime} \mathrm{N}, 121^{\circ} 30^{\prime} \mathrm{E}\right)$. Collected $1988-1989$ and submitted by Y. K. Chen and T.-K. Liu.

NTU-1107. LT770821B

Coral (aragonite $>95 \%$ ) from reef Terrace II, $\sim 50 \mathrm{~m}$ asl.

NTU-1143. LT770819D

Coral (aragonite $>95 \%$ ) from reef Terrace I, $\sim 2 \mathrm{~m}$ asl.

NTU-1144. LT770819C

Coral (aragonite $>95 \%$ ) from reef Terrace II, $\sim 50 \mathrm{~m}$ asl.

NTU-1150. LT770820B

Shell fragments from recent beach sand, $\sim 1 \mathrm{~m}$ asl.

NTU-1168. LT770821C

Shell from a marine terrace deposit, $\sim 10 \mathrm{~m}$ asl.

NTU-1171. LT770824A

Coral (aragonite $>95 \%$ ) from reef Terrace I, $\sim 2 \mathrm{~m}$ asl.

NTU-1199. LT780729B

Coral (aragonite $>95 \%$ ) from reef Terrace II, $\sim 50 \mathrm{~m}$ asl.
$33,100 \pm 410$

$\delta^{13} C=-0.9 \%$

$6140 \pm 50$

$\delta^{13} C=-0.9 \%$

$33,900 \pm 450$

$\delta^{13} \mathrm{C}=-2.2 \%$

$1530 \pm 40$

$\delta^{13} \mathrm{C}=+2.2 \%$

$3650 \pm 50$

$\delta^{13} \mathrm{C}=+2.2 \%$

$\mathbf{5 9 2 0} \pm \mathbf{5 0}$

$\delta^{13} C=-1.6 \% 0$

$49,210 \pm 2750$

$\delta^{13} C=-2.6 \% 0$

Comment (T.-K.L.): This age is distinguishable from background based on a $2 \sigma$ criterion.

NTU-1202. LT780729X

$5060 \pm 40$

Calcareous crust $\sim 2 \mathrm{~m}$ asl from a sea cave.

$\delta^{13} C=+2.6 \%$

NTU-1203. LT780730

Coral from reef Terrace I.

$5110 \pm 50$

NTU-1204. LT780728H

$\delta^{13} C=-0.9 \%$

Coral from reef Terrace I.

$6440 \pm 50$

$\delta^{13} C=+0.9 \%$

NTU-1207. LT780728D

$2400 \pm 40$

Coral from reef, $20-30 \mathrm{~m}$ asl.

$\delta^{13} \mathrm{C}=0.0 \%$ 
NTU-1221. LT780728C

$2120 \pm 40$

Coral from reef, $30 \mathrm{~m}$ asl.

$\delta^{13} \mathrm{C}=-1.1 \%$

Comment (Y.K.C.): Terrace I formed during the Holocene; Terrace II formed 30,000-50,000 yr BP.

NTU-1121. Tulan 001

$7680 \pm 60$

$\delta^{13} C=-27.6 \%$

Wood from Tulan Village, Taitung County $\left(22^{\circ} 51^{\prime} \mathrm{N}, 121^{\circ} 10^{\prime} \mathrm{E}\right)$, from yellowish-gray mud zone. Collected 1988-1989 and submitted by M. L. Hsieh.

Comment (P.M.C.): Dates submarine sliding.

Tainan

Tainan Tableland Series

Samples from within the city limits of Tainan, Tainan County, $\left(22^{\circ} 57^{\prime}-23^{\circ} 00^{\prime} \mathrm{N}, 121^{\circ} 11^{\prime}-18^{\prime} \mathrm{E}\right)$, from sandy silt of the Tainan Formation. Collected 1989-1990 and submitted by D. J. Wu and T.-K. Liu, National Taiwan University.

NTU-1147. CC540

Wood from tidal channel, $5.4 \mathrm{~m}$ below surface.

$\mathbf{5 8 4 0} \pm \mathbf{5 0}$
$\delta^{13} C=-25.0 \% 0$

NTU-1149. CC770

$5930 \pm 150$

Wood fragments, $7.7 \mathrm{~m}$ below surface.

$\delta^{13} C=-23.4 \%$

NTU-1153. CC1030

$7140 \pm 150$

Wood fragments, $10.3 \mathrm{~m}$ below surface.

$\delta^{13} \mathrm{C}=-25.6 \%$

NTU-1159. DM200

Shell, $2 \mathrm{~m}$ below surface.

$\mathbf{5 2 8 0} \pm \mathbf{5 0}$
$\delta^{13} C=+0.1 \%$

NTU-1184. CS780

$7210 \pm 60$

Shell, $7.8 \mathrm{~m}$ below surface.

$\delta^{13} C=-0.1 \%$ 。

NTU-1190. CK400A

$\mathbf{5 8 3 0} \pm \mathbf{5 0}$

Shell, $4 \mathrm{~m}$ below surface.

$\delta^{13} C=-1.4 \%$ 。

NTU-1214. GC250

$1710 \pm 40$

Shell, $0.5-2.5 \mathrm{~m}$ below surface.

$\delta^{13} C=-0.3 \%$

NTU-1254. SM500

$4800 \pm 30$

Shell, $5 \mathrm{~m}$ below surface.

$\delta^{13} C=+0.6 \%$ 
NTU-1256. FC400

$4690 \pm 50$

Shell, $4 \mathrm{~m}$ below surface.

Comment (T.-K.L.): Dates the range of deposition. The previous range was 6500-5000 BP (Lin 1969).

\section{Zinte Village Series}

Shell from Zinte Village, Tainan County $\left(22^{\circ} 57^{\prime} \mathrm{N}, 120^{\circ} 14^{\prime} \mathrm{E}\right)$, from the Tainan Formation. Collected 1989 and submitted by D. J. Wu and T.-K. Liu.

NTU-1248. 2T-1

$5510 \pm 30$

Oyster shell from sandy soil.

NTU-1262. JDC15

$\mathbf{5 3 0 0} \pm \mathbf{5 0}$

From a mud zone, $15-15.5 \mathrm{~m}$ below surface.

NTU-1269. JDC51

$8850 \pm 80$

From muddy silt, $51-51.5 \mathrm{~m}$ below surface.

Comment (T.-K.L.): Dates indicate continuous deposition during the Holocene.

\section{Kuanmiao Village Series}

Sample from Kuanmiao Village, Tainan County $\left(23^{\circ} 00^{\prime} \mathrm{N}, 120^{\circ} 16^{\prime} \mathrm{E}\right)$. Collected 1989 and submitted by Y. K. Chen and T.-K. Liu.

NTU-1192. 780522A

$34,080 \pm 450$

$\delta^{13} C=-26.0 \%$

Carbonized wood from the contact zone between the Tainan and Liushuang Formations.

NTU-1194. NY780811

$5840 \pm 50$

Shell fragments from inland gulf deposits.

$\delta^{13} C=-2.3 \%$

Comment (T.-K.L.): Dates confirm an unconformity between the Taiwan and Liushuang Formations.

NTU-1208. Paiho 813A

$12,250 \pm 70$

$\delta^{13} C=-29.4 \%$

Driftwood from Paiho Village, Tainan County, from river terrace deposits. Collected 1989 and submitted by Y. K. Chen.

NTU-1065. LPM-SKOUP

$40,450 \pm 780$

$\delta^{13} C=-25.0 \%$

Peat from Tsochen Village, Tainan County $\left(23^{\circ} 04^{\prime} \mathrm{N}, 120^{\circ} 24^{\prime} \mathrm{E}\right)$, from terrace deposits above bedrock. Collected 1978 and submitted by P. M. Liew.

Comment (P.M.L.): Date indicates this area emerged during the last glacial stage. 
NTU-1146. LSB

$>\mathbf{5 1 , 3 0 0}$

$\delta^{13} \mathrm{C}=-27.3 \%$

Wood from Liushuang Village, Tainan County, from the Liushuang Formation. Collected 1989 and submitted by Y. K. Chen.

NTU-1086. MU-02

$21,700 \pm 110$

$\delta^{13} C=-1.5 \%$

Shell from Liuchia Village, Tainan County $\left(23^{\circ} 21^{\prime} \mathrm{N}, 120^{\circ} 20^{\prime} \mathrm{E}\right)$, from the middle of the Tainan Formation. Collected 1989 and submitted by D. J. Wu.

NTU-1116. CWC780131A

$990 \pm 40$

$\delta^{13} \mathrm{C}=-26.2 \%$

Driftwood from Yuching Village, Tainan County $\left(23^{\circ} 13^{\prime} \mathrm{N}, 120^{\circ} 28^{\prime} \mathrm{E}\right)$, from alluvial deposits at lowest terrace. Collected 1989 and submitted by Y. K. Chen and T.-K. Liu.

NTU-1152. Tungshan

$9120 \pm 80$

$\delta^{13} C=-13.9 \%$

Peat from Tingshan Village, Tainan County $\left(22^{\circ} 15^{\prime} \mathrm{N}, 120^{\circ} 58^{\prime} \mathrm{E}\right)$, from alluvial deposits. Collected 1989 and submitted by Y. K. Chen.

\section{Pingtung}

\section{Hengchun Village Series}

Samples included shell, coral and other calcareous matter, from Hengchun Village, Pingtung County $\left(21^{\circ} 55^{\prime}-22^{\circ} 04^{\prime} \mathrm{N}, 120^{\circ} 41^{\prime}-120^{\circ} 51^{\prime} \mathrm{E}\right)$. Collected 1988 to 1989 and submitted by C. H. Wang and T.-K. Liu.

NTU-1064. HC1702

$40,900 \pm 800$

$\delta^{13} \mathrm{C}=+0.1 \%$

NTU-1089. HC1301

$36,100 \pm 450$

$\delta^{13} C=-2.0 \%$

NTU-1133. HC2301

$6270 \pm 50$

$\delta^{13} C=-1.6 \%$

NTU-1134. HC2304

$2530 \pm 40$

$\delta^{13} \mathrm{C}=-1.0 \%$

NTU-1135. HC2502

$\mathbf{5 8 4 0} \pm \mathbf{5 0}$

$\delta^{13} C=-0.7 \%$

NTU-1136. HC2503

$1390 \pm 40$

$\delta^{13} C=+0.5 \%$

NTU-1140. HC2504

$2850 \pm 40$

$\delta^{13} C=+0.5 \%$

NTU-1141. HC2505

$2160 \pm 40$

$\delta^{13} C=+0.1 \% 0$

NTU-1161. Hengchung

$4950 \pm 50$

$\delta^{13} C=+2.6 \% 0$ 
NTU-1165. Hengchung

$\mathbf{2 9 9 0} \pm \mathbf{5 0}$

$\delta^{13} C=+0.9 \%$

NTU-1166. Hengchung

$\mathbf{5 1 2 0} \pm \mathbf{5 0}$

$\delta^{13} C=+2.1 \%$

NTU-1173. Hengchung

$2460 \pm 50$

$\delta^{13} C=-0.1 \%$

Comment (C.H.W.): NTU-1165 is a mixture of marine and freshwater mollusks; the result is not valid for interpretation. Others are in-situ corals. Altitudes of dated corals and mollusks are compatible.

NTU-1215. HC781030A

$3220 \pm 40$

$\delta^{13} \mathrm{C}=+2.0 \%$

NTU-1216. HC781029B

$4870 \pm 40$

$\delta^{13} C=+1.5 \%$

NTU-1235. HC780101

$3530 \pm 40$

$\delta^{13} \mathrm{C}=-1.1 \%$

$>47,500$

NTU-1249. SLC780902A

$\delta^{13} C=-6.7 \%$

$3730 \pm 40$

NTU-1255. SLC780902B

$\delta^{13} C=-0.9 \%$

Corals and calcareous materials. Collected 1989 and submitted by Y. K. Chen.

NTU-1160. 77051604

$>37,000$

$\delta^{13} \mathrm{C}=-7.7 \%$

Corals from reef, $75 \mathrm{~m}$ asl. Collected 1989 and submitted by P. M. Liew.

NTU-1183. MCH0845

$3640 \pm 50$

$\delta^{13} C=-0.9 \%$

Coral from Machou Village, Pingtung County $\left(22^{\circ} 00^{\prime} \mathrm{N}, 120^{\circ} 51^{\prime} \mathrm{E}\right)$, from lowest marine terrace of Chialosui, $4 \mathrm{~m}$ asl. Collected 1988 and submitted by C. H. Wang.

Comment (C.H.W.): Good quality of sample material, in living position.

NTU-1246. HK

$5660 \pm 50$

$\delta^{13} \mathrm{C}=+1.6 \%$

Shell from Pingtung County, in Checheng Village $\left(22^{\circ} 05^{\prime} \mathrm{N}, 120^{\circ} 40^{\prime} \mathrm{E}\right)$, from sandy clay. Collected 1989 and submitted by C. H. Wang.

Comment (C.H.W.): Shell fragments in Holocene strata, $12 \mathrm{~m}$ asl, deposited on the beach.

\section{NTU-1209. PT-2}

$10,000 \pm 70$

$\delta^{13} C=-22.5 \%$

Wood from Wantan Village, Pingtung County $\left(22^{\circ} 35^{\prime} \mathrm{N}, 120^{\circ} 24^{\prime} \mathrm{E}\right)$, from sand and gravel zone, 96 $\mathrm{m}$ below surface. Collected 1989 by Z. S. Lee, Taiwan Sugar Company.

Comment (Z.S.L.): Large driftwood sample collected during well-water drilling in gravelly aquifer. 


\section{Hualien, Taiwan}

\section{Fengpin Village Series}

Samples from Fengpin Village, Hualien County $\left(23^{\circ} 29-35^{\prime} \mathrm{N}, 121^{\circ} 25-30^{\prime} \mathrm{E}\right)$. Collected 1989 and submitted by M. Y. Hsu, M. L. Hsieh and P. M. Liew.

NTU-1130. GP78-1

$3570 \pm 40$

Coral from a reef.

NTU-1157. GP78-2

Coral from a reef.

NTU-1158. GP-3

Coral from a reef.

Comment (M.Y.H.): Ages are as expected.

\section{NTU-1191. PALIWAN}

Wood from gray mud.

NTU-1239. Hsinshe 006

Wood from offshore deposits.

NTU-1252. Hsinshe 008

Wood from nearshore deposits, $15-20 \mathrm{~m}$ asl overlying volcanic bedrock.

Comment (P.M.L.): NTU-1239 corresponds with Shihtiping; NTU-1252 is much younger, confirming the alluvial fan environment of deposition.

\section{Hsiulien Village Series}

Wood from Hsiulien Village, Hualien County $\left(24^{\circ} 00^{\prime}-20^{\prime} \mathrm{N}, 121^{\circ} 20^{\prime}-40^{\prime} \mathrm{E}\right)$. Collected 1989 and submitted by T. H. Liu and T. M. Wang.

NTU-1185. L002

$2480 \pm 40$

Wood from a sand and pebble zone.

NTU-1228.

$3140 \pm 60$

Wood from a river terrace.

NTU-1243.

$3200 \pm 80$

Wood from a river terrace. 
NTU-1054. LPM-A

Wood from Shoufong Village, Hualien County $\left(23^{\circ} 52^{\prime} \mathrm{N}, 121^{\circ} 30^{\prime} \mathrm{E}\right)$, from the top of marine deposits, $3 \mathrm{~m}$ below surface. Collected 1988 by M. L. Hsieh; submitted by P. M. Liew.

NTU-1074. WSP

$4610 \pm 50$

$\delta^{13} C=-0.7 \%$

Calcareous matter from Hualien County. Collected 1988 and submitted by S. H. Weng, National Taiwan University.

NTU-1097. SL-4

$$
\begin{array}{r}
100.7 \pm 0.8 \mathrm{pMC} \\
\delta^{13} C=-26.3 \% \circ
\end{array}
$$

Organic matter from Shuilien Village, Hualien County $\left(23^{\circ} 47^{\prime} \mathrm{N}, 121^{\circ} 34^{\prime} \mathrm{E}\right)$, from a reef. Collected 1989 and submitted by M. C. Huang, Industrial Research Institute.

NTU-1128. 78012601

$1770 \pm 40$ $\delta^{13} C=+0.5 \%$

Coral from Chinpu Village, Hualien County $\left(23^{\circ} 27^{\prime} \mathrm{N}, 121^{\circ} 29^{\prime} \mathrm{E}\right)$, from a reef. Collected 1989 and submitted by C. H. Wang.

NTU-1186. 770226B

$6470 \pm 50$

$\delta^{13} C=-2.5 \%$

Coral from Milun Village, Hualien County $\left(24^{\circ} 01^{\prime} \mathrm{N}, 121^{\circ} 38^{\prime} \mathrm{E}\right)$, from raised reef terrace at $20 \mathrm{~m}$ asl. Collected 1988 and submitted by T.-K. Liu.

Comment (T.-K.L.): Good sample quality, but date is much older than previous dates of the same terrace (Lin 1969; Konishi, Omura and Kinura 1968).

Penghu

NTU-1148. PH770710

$4190 \pm 50$

$\delta^{13} \mathrm{C}=+0.4 \%$

Fresh coral from Chinglo Village, Penghu County $\left(23^{\circ} 36^{\prime} \mathrm{N}, 119^{\circ} 17^{\prime} \mathrm{E}\right)$, from a raised beach sand bed, $\sim 3 \mathrm{~m}$ asl. Collected 1988 and submitted by T.-K. Liu and Y. K. Chen.

Comment (T.-K.L.): The date represents the youngest and most pervasive raised marine terrace of Penghu and probably implies a higher eustatic sea level.

\section{Chihkan Village Series}

Fresh coral from Chihkan Village, Penghu County $\left(23^{\circ} 40^{\prime} \mathrm{N}, 119^{\circ} 16^{\prime} \mathrm{E}\right)$, from a calcareous sand layer $\sim 2.5 \mathrm{~m}$ asl. Collected 1989 and submitted by T.-K. Liu and Y. K. Chen.

NTU-1175. CK-01A

Coral fragments.

NTU-1176. CK-02C

Coral fragments. $\mathbf{4 3 4 0} \pm \mathbf{5 0}$ $\delta^{13} \mathrm{C}=+0.8 \%$

$4490 \pm 50$

$\delta^{13} C=+2.1 \%$

Comment (T.-K.L.): Dates agree well with NTU-1148 from the same marine terrace level in Chinglo. 
NTU-1177. DGA

$760 \pm 40$

$\delta^{13} C=+1.5 \%$

Coral fragments from Tungchi Village, Penghu County $\left(23^{\circ} 15^{\prime} \mathrm{N}, 119^{\circ} 20^{\prime} \mathrm{E}\right)$. Collected 1989 and submitted by T.-K. Liu and Y. K. Chen.

Comment (T.-K.L.): This sample is not in-situ, as seems likely from its elevation $(\sim 50 \mathrm{~m}$ asl) and developed cultivation.

NTU-1178. BK-A

$4510 \pm 50$

$\delta^{13} \mathrm{C}=+1.2 \%$

Fresh coral from Paikeng Village, Penghu County $\left(23^{\circ} 35^{\prime} \mathrm{N}, 119^{\circ} 17^{\prime} \mathrm{E}\right)$. Collected 1989 and submitted by T.-K. Liu and Y. K. Chen.

Comment (T.-K.L.): Date is compatible with dates from the same terrace in Chinglo and Chihkan.

\section{Kupoyu Series}

Shell and coral fragments from Kupoyu Island, Penghu County $\left(23^{\circ} 45^{\prime} \mathrm{N}, 119^{\circ} 14^{\prime} \mathrm{E}\right)$ from calcareous sand. Collected 1989 and submitted by T.-K. Liu and Y. K. Chen.

\section{NTU-1179. GP-A}

NTU-1182. GP-B

$$
\begin{array}{r}
470 \pm 50 \\
\delta^{13} C=+1.6 \% 0 \\
900 \pm 40 \\
\delta^{13} C=+2.5 \%
\end{array}
$$

Comment (T.-K.L.): Younger than expected. The sample was dissolved during pretreatment to half of its original weight.

\section{NTU-1180. FK-A}

$1890 \pm 50$

$\delta^{13} C=+2.2 \%$

Shell from Fengkuei Village, Penghu County $\left(23^{\circ} 31^{\prime} \mathrm{N}, 119^{\circ} 19^{\prime} \mathrm{E}\right)$. Collected 1989 and submitted by T.-K. Liu and Y. K. Chen.

Comment (T.-K.L.): Dates coastal part of the lowest raised marine terrace $\sim 1.5 \mathrm{~m}$ asl in Fengkuei.

NTU-1181. NU-A

$5460 \pm 50$

$\delta^{13} \mathrm{C}=-1.1 \%$

Calcareous sandy carbonaceous materials from Niaoyuug Village, Penghu County $\left(23^{\circ} 40^{\prime} \mathrm{N}, 119^{\circ}\right.$ 19'E). Collected 1989 and submitted by T.-K. Liu and Y. K. Chen.

Comment (T.-K.L.): Dates sand dune $\sim 10 \mathrm{~m}$ asl.

NTU-1220. LV-K

Shell from Lungmen Village, Penghu County $\left(23^{\circ} 35^{\prime} \mathrm{N}, 119^{\circ} 20^{\prime} \mathrm{E}\right)$, from soil zone overlying basalt. Collected 1989 and submitted by C. H. Wang.

Comment (C. H.W.): $\sim 10 \mathrm{~m}$ asl, probably transported by ancient human activities. 
Nantou

NTU-1109. 88026

$42,500 \pm 950$

$\delta^{13} C=-29.9 \%$

Wood from Yuchih Village, Nantou County $\left(23^{\circ} 55^{\prime} \mathrm{N}, 120^{\circ} 56^{\prime} \mathrm{E}\right)$, from a white clay zone. Collected 1988 and submitted by C. M. Hsu.

Comment (C.M.H.): Date is younger than expected.

NTU-1164. Teng-120

Carbonized wood from Tsaoyun Village, Nantou County $\left(23^{\circ} 58^{\prime} \mathrm{N}, 120^{\circ} 41^{\prime} \mathrm{E}\right)$. Collected 1988 and submitted by S. Y. Teng, National Taiwan University.

Comment (S.Y.T.): Sample is from fluvial deposits of the Toukoshan Formation. Date confirms previous estimate.

NTU-1196. PL-1

$$
\delta^{13} C=\begin{array}{r}
\mathbf{> 5 0 , 0 0 0} \\
-29.3 \% \circ
\end{array}
$$

Carbonized wood from Puli Village, Nantou County $\left(24^{\circ} 00^{\prime} \mathrm{N}, 120^{\circ} 00^{\prime} \mathrm{E}\right)$, from a muddy sand zone, $5 \mathrm{~m}$ below surface. Collected 1988 and submitted by Y. K. Chen and T.-K. Liu.

Kaohsiung

NTU-1167. CKB780722

$4410 \pm 50$

$\delta^{13} \mathrm{C}=-5.8 \%$

Carbonaceous matter from Tienliao Village, Kaohsiung County $\left(22^{\circ} 53^{\prime} \mathrm{N}, 120^{\circ} 21^{\prime} \mathrm{E}\right)$, from the bottom of transgressive sand. Collected 1989 and submitted by Y. K. Chen and T.-K. Liu.

NTU-1104. KS12-1-16

$3470 \pm 70$

$\delta^{13} C=-0.7 \%$

Foraminifera (Globigerina sp) from offshore Kaohsiung $\left(22^{\circ} 30^{\prime} \mathrm{N}, 119^{\circ} 30^{\prime} \mathrm{E}\right)$, from the $279-280 \mathrm{~cm}$ interval of core. Collected 1988 and submitted by C. H. Wang.

Comment (C.H.W.): Although the sample was small, the calculated sedimentation rate of $80.7 \mathrm{~cm}$ $1000 \mathrm{yr}^{-1}$ agrees with previous studies.

Miaoli

Paishatun Village Series

Calcareous matter from Paishatun Village, Miaoli County $\left(24^{\circ} 30^{\prime} \mathrm{N}, 120^{\circ} 45^{\prime} \mathrm{E}\right)$. Collected 1988 and submitted by $\mathrm{C}$. H. Wang.

NTU-1119. 880921A

$30,900 \pm 350$

Altitude $\sim 10 \mathrm{~m}$.

$\delta^{13} \mathrm{C}=+1.1 \%$

NTU-1120. $880921 B$

$36,700 \pm 600$

Altitude $\sim 12 \mathrm{~m}$.

$\delta^{13} \mathrm{C}=+1.2 \%$ 
NTU-1123. 771116

$44,900 \pm 1600$

Altitude $\sim 15 \mathrm{~m}$.

$\delta^{13} \mathrm{C}=+1.3 \%$

Shell (Pecten byorituensis) from Houlun Village, Miaoli County $\left(24^{\circ} 30^{\prime} \mathrm{N}, 120^{\circ} 40^{\prime} \mathrm{E}\right)$. Collected 1988 and submitted by C. H. Wang.

Comment (C.H.W.): Dates have a positive correlation with their altitudes.

NTU-1193. LUT

$13,300 \pm 80$

$\delta^{13} C=-28.0 \%$

Wood from Sanyi Village, Miaoli County $\left(24^{\circ} 20^{\prime} \mathrm{N}, 120^{\circ} 46^{\prime} \mathrm{E}\right)$, from an organic zone in muddy deposits. Collected 1989 and submitted by P. S. Chang, Chungsin Engineering Consulting Group.

Ilan

NTU-1266. TC-1

$6480 \pm 50$

$\delta^{13} \mathrm{C}=+1.0 \%$

Fresh coral from Toucheng Village, Ilan County $\left(24^{\circ} 57^{\prime} \mathrm{N}, 120^{\circ} 54^{\prime} \mathrm{E}\right)$, from a newly emerged wavecut beach. Collected 1990 and submitted by M. Y. Hsu.

Comment (M.Y.H.): Age is as expected, and implies a relatively low rate of crustal uplift.

NTU-1275.

$$
\delta^{13} C=\begin{array}{r}
>46,000 \\
-28.2 \% 0
\end{array}
$$

Wood from Yuanshan Village, Ilan County $\left(24^{\circ} 45^{\prime} \mathrm{N}, 121^{\circ} 38^{\prime} \mathrm{E}\right)$, from contact of mud-conglomerate zone. Collected 1990 and submitted by H. Y. Chen.

Comment (H.Y.C.): Dates ancient filled lake.

Taoyuan

NTU-1079. 770412A

$2510 \pm 40$

$\delta^{13} C=-28.0 \%$

Carbonized wood from Nankan Village, Taoyuan County $\left(25^{\circ} 00^{\prime} \mathrm{N}, 121^{\circ} 22^{\prime} \mathrm{E}\right)$, from alluvial deposits. Collected 1988 and submitted by T.-K. Liu and Y. K. Chen.

\section{Chiayi}

NTU-1112. UCH-1

$40,200 \pm 960$

$\delta^{13} C=-29.5 \%$

Wood from Minhsiung Village, Chiayi County $\left(23^{\circ} 33^{\prime} \mathrm{N}, 120^{\circ} 25^{\prime} \mathrm{E}\right)$, from a mudstone layer. Collected 1989 and submitted by M. C. Huang.

Comment (M.C.H.): Sample was dated to study the activity of the Meishan fault. Collected from an artificial trench in an alluvial fan.

\section{NTU-1231. Well-1}

$$
\begin{aligned}
& >50,000 \\
\delta^{13} C & =-24.9 \% 0
\end{aligned}
$$

Wood from Aoku Village, Chiayi County $\left(23^{\circ} 33^{\prime} \mathrm{N}, 120^{\circ} 20^{\prime} \mathrm{E}\right)$, from an exploration well, at the $165-$ 170-m interval. Collected 1989 and submitted by P. M. Liew.

Comment (P.M.L.): Older than expected; more dates are needed to confirm this result. 


\section{Other Localities}

Kinmen Island

NTU-1250. KM89-24

$42,600 \pm 770$

$\delta^{13} C=-25.7 \%$

Wood from Kinmen Island $\left(24^{\circ} 20^{\prime} \mathrm{N}, 118^{\circ} 00^{\prime} \mathrm{E}\right)$. Collected 1989 and submitted by C. M. Wang.

NTU-1267. KM-TD

$680 \pm 40$

$\delta^{13} C=+1.6 \%$

Shell fragments from Tati Village, Kinmen Island $\left(2^{\circ} 32^{\prime} \mathrm{N}, 119^{\circ} 30^{\prime} \mathrm{E}\right)$, from a marine terrace. Collected 1990 and submitted by C. M. Wang.

NTU-1271. 040105-2

$2660 \pm 100$

$\delta^{13} \mathrm{C}=-0.2 \%$

Shell from Kinmen Island, in Kinni Village $\left(24^{\circ} 27^{\prime} \mathrm{N}, 118^{\circ} 24^{\prime} \mathrm{E}\right)$, found at a low tidal zone. Collected 1990 and submitted by P. M. Liew.

NTU-1273. KMKS

$4280 \pm 40$

$\delta^{13} \mathrm{C}=+2.4 \%$

Shell from Kinmen Island $\left(24^{\circ} 32^{\prime} \mathrm{N}, 119^{\circ} 30^{\prime} \mathrm{E}\right)$. Collected 1990 and submitted by C. M. Wang.

Southeastern Offshore of Taiwan

\section{H B K Series}

Forams ( $>63 \mu \mathrm{m}$ fraction) from off the southeastern Taiwan coast $\left(22^{\circ} 00-43^{\prime} \mathrm{N}, 121^{\circ} 10-30^{\prime} \mathrm{E}\right)$, from marine-core sediments. Collected 1988-1989 and submitted by M. P. Chen.

NTU-1245. HBK-1

$37,330 \pm 2490$

At $20 \mathrm{~m}$ depth. $\delta^{13} \mathrm{C}=+0.4 \%$

NTU-1263. HBK-4

$1200 \pm 80$

At $0-5 \mathrm{~cm}$ depth. $\delta^{13} C=+0.4 \% 0$

NTU-1274. HBK-5

$2000 \pm 90$

At $12.5-17.5-\mathrm{cm}$ depth.

Comment (M.P.C.): Samples are from piston core OR-170-7. HBK-4 and -5 are from box core OR216-17B.

\section{OR-102-6 Series}

Foram ooze from Lanshu Ridge $\left(22^{\circ} 17^{\prime} \mathrm{N}, 121^{\circ} 28^{\prime} \mathrm{E}\right)$, from marine core OR-102-6. Collected 1988-1989 and submitted by M. P. Chen.

NTU-1055. OR-102-6-1

At $162-165 \mathrm{~cm}$ depth.

NTU-1125. OR-102-6-2

At 80-82 cm depth.
$35,960 \pm 1640$

$\delta^{13} \mathrm{C}=+0.8 \%$

$16,500 \pm 460$

$\delta^{13} C=+1.4 \%$ 
NTU-1126. OR-102-6-3

$9040 \pm 150$

At $0-2 \mathrm{~cm}$ depth.

$\delta^{13} \mathrm{C}=+1.2 \%$

Comment (M.P.C.): The samples were highly concentrated in the foram tests, so the conventional bulk acidification method was still feasible.

\section{Philippine Islands}

\section{Batan Island Series}

Carbonized wood and fresh coral from Batan Island, The Philippines $\left(20^{\circ} 25^{\prime} \mathrm{N}, 122^{\circ} 56^{\prime} \mathrm{E}\right)$, from muddy volcanic ash. Collected 1989 and submitted by T. F. Lee, Institute of Earth Sciences.

NTU-1138. Typhoon-PF

NTU-1139. Mahatao

Fresh coral fragments.

$$
\begin{array}{r}
920 \pm 90 \\
\delta^{13} C=-29.6 \% \circ \\
102.1 \pm 0.7 \mathrm{pMC} \\
\delta^{13} C=+0.8 \% 0
\end{array}
$$

\section{SAMPLES OF THE INTERNATIONAL COLLABORATIVE STUDY}

The Radiocarbon Laboratory of National Taiwan University has participated in all three stages of the International Collaborative Study (ICS) conducted at the University of Glasgow (Scott et al. $1990 \mathrm{a}, \mathrm{b})$. Our lab code is 8 . We present here our results of the third and final stage.

\section{NTU-1095. ICS-8I}

$\mathbf{3 3 2 1} \pm \mathbf{5 0}$

Peat; ICS median age $=3375 \mathrm{BP} ;$ mean age $=3388 \mathrm{BP}$.

$\delta^{13} C=-26.8 \%$

NTU-1099. ICS-8L

$2203 \pm 50$

Wood; ICS calendar age $=221-240 \mathrm{BC}$, equivalent ${ }^{14} \mathrm{C}$ age $=2185 \mathrm{BP}$.

$\delta^{13} C=-25.0 \%$

NTU-1103. ICS-8M

$79 \pm 40$

Wood; ICS calendar age $=1841-1870 \mathrm{AD}$, equivalent ${ }^{14} \mathrm{C}$ age $=100 \mathrm{BP}$.

$\delta^{13} C=-25.8 \%$

NTU-1101. ICS-8N(=ICS 8L)

$2192 \pm 40$

Wood; ICS calendar age $=221-240 \mathrm{BC}$, equivalent ${ }^{14} \mathrm{C}$ age $=2185 \mathrm{BP}$.

$\delta^{13} C=-24.1 \%$

NTU-1102. ICS-8P

$279 \pm 40$

Wood; ICS calendar age $=1521-1550 \mathrm{AD}$, equivalent ${ }^{14} \mathrm{C}$ age $=290 \mathrm{BP}$.

$\delta^{13} C=-23.4 \%$

NTU-1075. ICS-8S(=ICS 8V)

$648 \pm 40$

Shell; ICS median age $=662 \mathrm{BP}$; mean age $=660 \mathrm{BP}$.

$\delta^{13} \mathrm{C}=+1.2 \%$

NTU-1084. ICS-8V

$662 \pm 50$

Algae; ICS median age $=662 \mathrm{BP} ;$ mean age $=660 \mathrm{BP}$. 


\section{REFERENCES}

Coleman, D. D. 1973 Illinois State Geological Survey radiocarbon dates IV. Radiocarbon 15(1): 75-85.

Konishi, K., Omura, A. and Kimura, T. $1968 \mathrm{U}^{234}-\mathrm{Th}^{230}$ dating of some late Quaternary coralline limestones from southern Taiwan (Formosa). Geology and Paleontology of Southeast Asia 5: 211-224.

Lin, C. C. 1969 Holocene geology of Taiwan. Acta Geologica Taiwanica 13: 83-126.

Scott, E. M., Aitchison, T. C., Harkness, D. D., Cook, G. T. and Baxter, M. S. 1990a An overview of all three stages of the International Radiocarbon Intercomparison. In Scott, E. M., Long, A. and Kra, R. S., eds., Pro-

ceedings of the International Workshop on Intercomparison of Radiocarbon Laboratories. Radiocarbon 32(3): 309-319.

Scott, E. M., Baxter, M. S., Aitchison, T. C., Harkness, D. D. and Cook, G. T. 1990b An overview of some interlaboratory studies. In Scott, E. M., Long, A. and Kra, R. S., eds., Proceedings of the International Workshop on Intercomparison of Radiocarbon Laboratories. Radiocarbon 32(3): 259-265.

Stuiver, M. and Polach, H. A. 1977 Discussion: Reporting of ${ }^{14} \mathrm{C}$ data. Radiocarbon 19(3): 355-363. 